\title{
Assembly factors Rpf2 and Rrs1 recruit 5S rRNA and ribosomal proteins rpL5 and rpL11 into nascent ribosomes
}

\author{
Jingyu Zhang, ${ }_{1}^{1}$ Piyanun Harnpicharnchai, ${ }^{1}$ Jelena Jakovljevic, ${ }^{1}$ Lan Tang, ${ }^{1}$ Yurong Guo, ${ }^{2}$ \\ Marlene Oeffinger, ${ }^{3}$ Michael P. Rout, ${ }^{3}$ Shawna L. Hiley, ${ }^{4}$ Timothy Hughes, ${ }^{4}$ and \\ John L. Woolford Jr. ${ }^{1,5}$ \\ ${ }^{1}$ Department of Biological Sciences, Carnegie Mellon University, Pittsburgh, Pennsylvania 15213, USA; ${ }^{2}$ Division of \\ Pulmonary and Critical Care Medicine, School of Medicine, John Hopkins University, Baltimore, Maryland 21224, USA; \\ ${ }^{3}$ Rockefeller University, New York, New York 10021, USA; ${ }^{4}$ Banting and Best Department of Medical Research, University \\ of Toronto, Toronto, Ontario M5S 3E1, Canada
}

\begin{abstract}
More than 170 proteins are necessary for assembly of ribosomes in eukaryotes. However, cofactors that function with each of these proteins, substrates on which they act, and the precise functions of assembly factors-e.g., recruiting other molecules into preribosomes or triggering structural rearrangements of pre-rRNPs-remain mostly unknown. Here we investigated the recruitment of two ribosomal proteins and $5 \mathrm{~S}$ ribosomal RNA (rRNA) into nascent ribosomes. We identified a ribonucleoprotein neighborhood in preribosomes that contains two yeast ribosome assembly factors, Rpf2 and Rrs1, two ribosomal proteins, rpL5 and rpL11, and $5 S$ rRNA. Interactions between each of these four proteins have been confirmed by binding assays in vitro. These molecules assemble into $90 \mathrm{~S}$ preribosomal particles containing $35 \mathrm{~S}$ rRNA precursor (pre-rRNA). Rpf2 and Rrs1 are required for recruiting rpL5, rpL11, and 5S rRNA into preribosomes. In the absence of association of these molecules with pre-rRNPs, processing of 27SB pre-rRNA is blocked. Consequently, the abortive $66 \mathrm{~S}$ pre-rRNPs are prematurely released from the nucleolus to the nucleoplasm, and cannot be exported to the cytoplasm.
\end{abstract}

[Keywords: 5S rRNA; rpL5; rpL11; yeast preribosomes; ribosome assembly subcomplexes]

Supplemental material is available at http://www.genesdev.org

Received May 9, 2007; revised version accepted August 21, 2007.

In eukaryotes, 79 ribosomal proteins associate with ribosomal RNA (rRNA) to produce 40S and 60S ribosomal subunits (Woolford and Warner 1991). Three of the four rRNAs in mature ribosomes are derived from the 35S45S rRNA precursor (pre-rRNA) transcribed by RNA polymerase I, while the fourth rRNA, 5S rRNA, is transcribed from separate genes by RNA polymerase III. The 35S-45S primary transcript is packaged into a 90S ribonucleoprotein particle (RNP), together with a subset of assembly factors and ribosomal proteins. Subsequent steps trigger folding, modification, and processing of prerRNAs and association of additional assembly factors and ribosomal proteins in $43 \mathrm{~S}$ and $66 \mathrm{~S}$ assembly intermediates. These pre-rRNPs undergo further maturation in the nucleolus, nucleoplasm, and then cytoplasm to form functional 40S and 60S ribosomal subunits, respectively (Fig. 1A; Fatica and Tollervey 2002; FromontRacine et al. 2003; Raué 2003; Granneman and Baserga

\footnotetext{
${ }^{5}$ Corresponding author.
}

E-MAIL jw17@andrew.cmu.edu; FAX (412) 268-7129.

Article is online at http://www.genesdev.org/cgi/doi/10.1101/gad.1569307.
2004). Preribosomal particles in the assembly pathway are distinguished by the presence of successive prerRNA processing intermediates (Fig. 1A). However, it is not clear into which of the consecutive preribosomes $5 \mathrm{~S}$ rRNA and each ribosomal protein are incorporated, which assembly factors are required to recruit these molecules, or how they do so. Furthermore, the mechanisms by which constituents of nascent ribosomes facilitate folding, processing, and modification of pre-rRNAs remain elusive.

$5 S$ rRNA is essential for maturation of preribosomes and for the function of mature ribosomes (Van Ryk et al. 1992; Dechampesme et al. 1999; Kiparisov et al. 2005). Steitz and coworkers defined a pathway of assembly of 5S rRNA into ribosomes in HeLa cells. Newly synthesized 5S pre-rRNA binds transiently to the La protein (Rinke and Steitz 1982; Yoo and Wolin 1994). Following 3 '-end maturation, 5S rRNA binds to ribosomal protein rpL5, then assembles into ribosomes (Steitz et al. 1988). In yeast, extraribosomal 5S rRNA was found in a complex with rpL5, indicating that yeast rpL5 also associates with 5S rRNA before incorporation into preribo- 

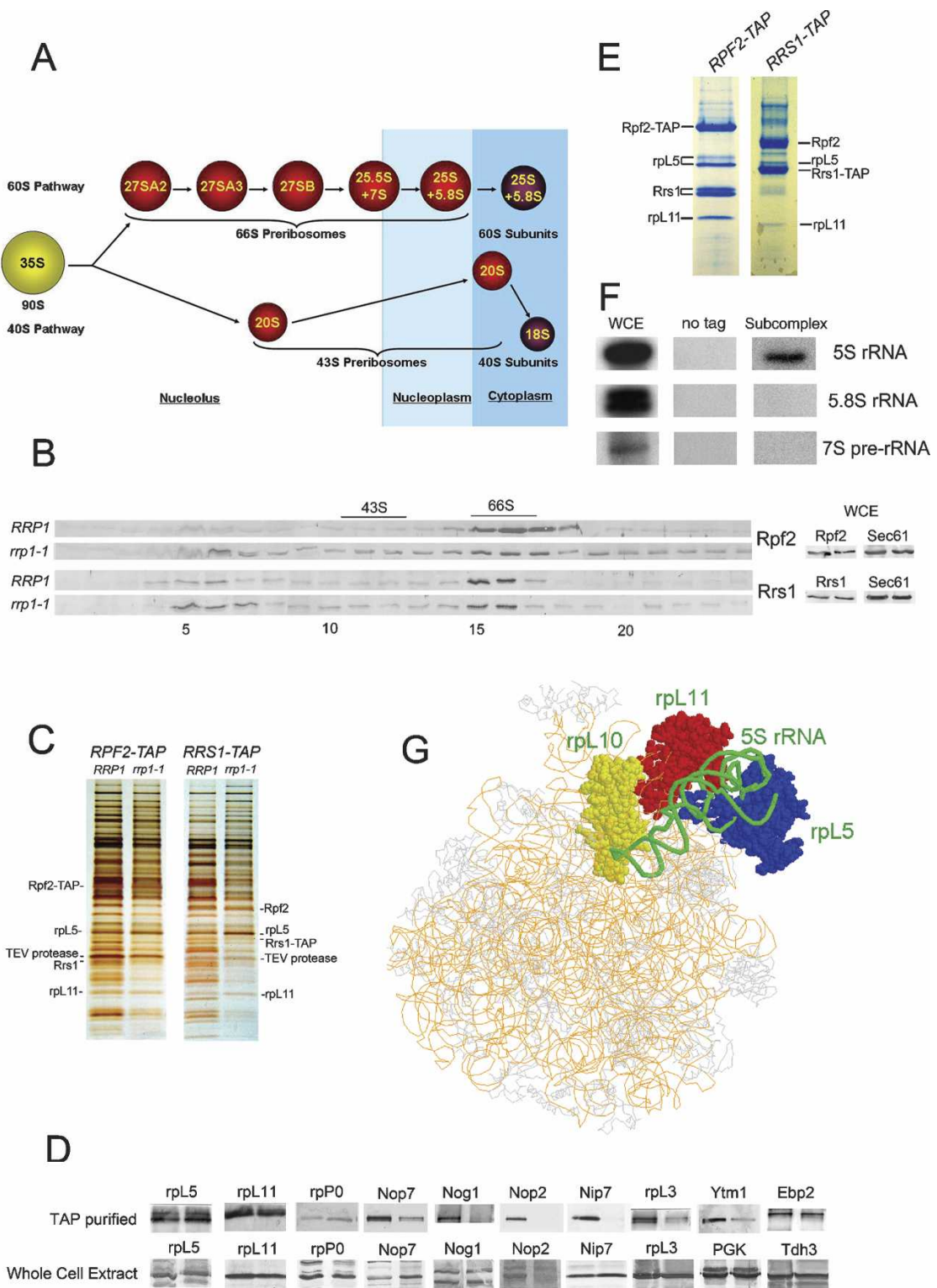

Figure 1. Identification and purification of a subcomplex containing $5 \mathrm{~S}$ rRNA, ribosomal proteins rpL5 and rpL11, and assembly factors Rpf2 and Rrs1. (A) The pre-rRNP maturation pathway in Saccharomyces cerevisiae. The $90 \mathrm{~S}$ pre-rRNP containing $35 \mathrm{~S}$ pre-rRNA and a subset of ribosomal proteins and assembly factors is converted into $43 \mathrm{~S}$ and $66 \mathrm{~S}$ precursor particles, then to mature $40 \mathrm{~S}$ and $60 \mathrm{~S}$ ribosomal subunits. Four consecutive $66 \mathrm{~S}$ pre-rRNPs contain $27 \mathrm{SA}_{2}$ $27 \mathrm{SA}_{3}, 27 \mathrm{SB}$, and 25.5 plus $7 \mathrm{~S}$ pre-rRNAs, respectively, plus ribosomal proteins and assembly factors. Ribosome biogenesis begins in the nucleolus and continues in the nucleoplasm; final steps occur in the cytoplasm. (B, left) Amounts of Rpf2 or Rrs1 sedimenting as a small complex near the top of gradients increase in the rrp1-1 mutant compared with wild-type cells. Wildtype yeast (JWY7087 and JWY7461) and temperature-sensitive rrp1-1 mutant yeast (JWY7089 and JWY7462) expressing TAPtagged Rpf2 or Rrs1 were grown at $25^{\circ} \mathrm{C}$ to $1.5 \times 10^{7}$ cells per milliliter and shifted for $5 \mathrm{~h}$ to $37^{\circ} \mathrm{C}$. Extracts from the wild-type (top) and rrp1-1 mutant (bottom) strains were fractionated on $7 \%-47 \%$ sucrose gradients, and amounts of Rpf2-TAP or Rrs1TAP proteins in each fraction were assayed by Western immunoblotting. Fractions containing $43 \mathrm{~S}$ and $66 \mathrm{~S}$ preribosomes are indicated. (Right) Amounts of Rpf2 and Rrs1 in whole-cell extracts remain the same in rrp1-1 mutant compared with wildtype cells. (C) Enrichment of the Rpf2-subcomplex in the rrp1-1 mutant compared with other preribosomal proteins. Wholecell extracts from the RRP1 and rrp1-1 strains were subjected to TAP using Rpf2TAP or Rrs1-TAP. Copurifying proteins were resolved by SDS-PAGE and stained with silver. Proteins enriched in mutant strains are labeled. $(D)$ Enrichment of proteins in TAP-purified samples does not result from changes in their amounts in whole-cell extracts. TAP-purified samples from the RPF2-TAP strains JWY7087 or JWY7089 $(t o p)$, and proteins from whole-cell extracts of the same strains (bottom), were subjected to Western blot analysis. Samples on the left in each pair are from wild-type $R R P 1$ cells and those on the right are from the rrp1-1 mutant. Proteins from equal amounts of cells were loaded in each lane. Similar results were obtained with the RRS1-TAP strain (data not shown). (E) The purified Rpf2 subcomplex contains Rpf2, Rrs1, rpL5, and rpL11. Fractions 5-7 pooled from the gradients of rrp1-1 extracts, shown in $B$, were subjected to TAP purification using either Rpf2-TAP or Rrs1-TAP. Purified proteins were resolved by SDS-PAGE, stained by Coomassie blue, and identified by mass spectrometry. (F) $5 \mathrm{~S}$ rRNA is present in the purified Rpf2 subcomplex. RNA was extracted from whole-cell extracts (left), affinity-purified samples from untagged strain (middle), and Rpf2 subcomplex purified from gradient fractions 5-7 (right), resolved by polyacrylamide gel electrophoresis, and assayed by Northern blotting using specific oligonucleotide probes. $(G)$ Docking of atomic models of ribosomal proteins and 5S rRNA into a $15 \AA$ resolution cryoelectron microscopy map of yeast $60 \mathrm{~S}$ ribosomal subunits (Spahn et al. 2001) demonstrates that rpL5 (blue), rpL10 (yellow), rpL11 (red), and 5S rRNA (green) are adjacent to one another in mature $60 \mathrm{~S}$ ribosomal subunits.

somes (Deshmukh et al. 1993). In Xenopus oocytes, 5S rRNA is synthesized before other constituents of ribosomes and is stored in the cytoplasm for long periods as 7S RNPs that contain transcription factor TFIIIA or as 42S RNPs containing the two thesaurin proteins and tRNAs (Picard and Wegnez 1979; Guddat et al. 1990; Viel et al. 1991). During vitellogenesis, 5S rRNA is released from these particles, associates with rpL5, and assembles into ribosomes. In bacteria, association of $5 \mathrm{~S}$ rRNA with ribosomes is dependent on ribosomal proteins L18 (homolog of eukaryotic rpL5), L5 (homolog of eukaryotic rpL11), and L25 (no obvious homolog) (Yu and Wittmann 
1973). These three proteins can bind to 5S rRNA independently of ribosomes and can associate in vitro with ribosome assembly intermediates (Gray et al. 1972; Spierer and Zimmermann 1978). However, it has not yet been determined whether the same pathway of assembly of $5 \mathrm{~S}$ rRNA into bacterial ribosomes occurs in vivo. In HeLa cells, 5S rRNA is first detected in 55S precursors to $60 S$ subunits, but exactly which precursors is unclear (Warner and Soeiro 1967). In yeast, expression of certain unstable mutant forms of yeast $5 \mathrm{~S}$ rRNA slows processing of $27 \mathrm{SB}$ pre-rRNA to mature $25 \mathrm{~S}$ and $5.8 \mathrm{~S}$ rRNAs and diminishes production of $60 \mathrm{~S}$ ribosomal subunits (Van Ryk et al. 1992; Dechampesme et al. 1999). Taken together, these results suggest that 5S rRNA assembles together with rpL5 into preribosomes at an intermediate step in particle maturation, possibly before 27SB prerRNA undergoes further processing. However, which assembly factors, if any, recruit 5S rRNA into pre-rRNPs, and into which assembly intermediates 5S rRNA is recruited, remain unknown.

How and when does each ribosomal protein join nascent ribosomes and what roles does each protein play in ribosome synthesis? In bacteria, in vitro reconstitution of ribosomes revealed a hierarchical pathway of assembly of ribosomal proteins with rRNA (Held et al. 1973). Whether this pathway is identical to that in vivo remains to be established. In contrast, there is only a lowresolution map for the pathway of ribosome assembly in eukaryotes. Kinetic labeling studies in yeast identified several ribosomal proteins that assemble into preribosomes at relatively early or late stages (Kruiswijk et al. 1978). Which assembly factors might recruit ribosomal proteins has not been determined, except in a few cases. Yeast ribosome assembly factor Rrb1 physically interacts with r-protein rpL3 and regulates its expression (Iouk et al. 2001), suggesting that Rrb1 might target rpL3 into preribosomes (Schaper et al. 2001). Assembly factor Sqt1 binds to rpL10p (Eisinger et al. 1997), and is thought to facilitate loading of rpL10 into preribosomes in the cytoplasm (West et al. 2005). The participation of each small subunit ribosomal protein in biogenesis of $40 \mathrm{~S}$ ribosomes, specifically maturation of $18 \mathrm{~S}$ rRNA and nuclear export of $43 \mathrm{~S}$ preribosomes, has been systematically examined. All essential ribosomal proteins of the small subunit except rpS7, rpS30, and rpS31 are required for pre-rRNP maturation at different intermediate steps. More interestingly, correlations between the timing of assembly and the timing of function were observed for some of these ribosomal proteins (Ferreira-Cerca et al. 2005), which emphasizes the importance of studying recruitment of ribosomal proteins and their functions in ribosome synthesis. In contrast, functions in ribosome assembly for most large subunit ribosomal proteins remain mostly unexplored except for rpL10, which is necessary for recycling of the export adaptor Nmd3 (West et al. 2005).

Here we identify a ribonucleoprotein neighborhood in preribosomes that contains two assembly factors, Rpf2 and Rrs1, two ribosomal proteins, rpL5 and rpL11, and 5S rRNA. We show that Rpf2 and Rrs1 are necessary for assembly of 5S rRNA, rpL5, and rpL11 into 90S preribo- somes containing $35 \mathrm{~S}$ pre-rRNA. In the absence of incorporation of these molecules into nascent ribosomes, preribosomal particles remain largely intact; however, 27SB pre-rRNA cannot efficiently undergo further processing. Consequently, these abortive 66S pre-rRNPs are prematurely released from the nucleolus to the nucleoplasm, and cannot be exported to the cytoplasm. Our characterization of the Rpf 2 neighborhood provides novel insights into when and how ribosomal proteins and 5S rRNA are delivered into nascent ribosomes.

\section{Results}

A subcomplex in preribosomes that contains ribosome assembly factors Rpf2 and Rrs1, ribosomal proteins $r p L 5$ and $r p L 11$, and $5 S$ rRNA

Previous yeast two-hybrid experiments revealed pairwise interactions between Rpf2, Rrs1, and rpL11 (Miyoshi et al. 2002; Morita et al. 2002). In mature yeast ribosomes, rpL11 is adjacent to 5S rRNA and rpL5 (Fig. 1G; Tsay et al. 1994; Spahn et al. 2001). Thus, it is possible that in ribosome assembly intermediates, 5S rRNA and rpL5 are close neighbors of Rpf2, Rrs1, and rpL11. To determine whether these five molecules form a neighborhood within preribosomal particles, we used a combined genetic and biochemical approach to purify subcomplexes from nascent ribosomes. We took advantage of the phenotype of the rrp1-1 mutant defective in ribosome biogenesis. When this temperature-sensitive mutant is shifted to the nonpermissive temperature, maturation of $60 \mathrm{~S}$ ribosomal subunits is blocked, $27 \mathrm{~S}$ prerRNA processing intermediates are degraded, and 66S pre-rRNPs fall apart into subcomplexes (Andrew et al. 1976; Harnpicharnchai et al. 2001; Horsey et al. 2004; Miles et al. 2005). This enables identification of neighborhoods that would normally form within assembling ribosomes, but dissociate from them when preribosomal particles in the rrp1-1 mutant disassemble upon shifting to nonpermissive temperature. Such subcomplexes would also be evident in the rrp1-1 mutant if they assembled outside of pre-rRNPs, but were unable to join nascent ribosomes when Rrpl is inactive.

Two initial results suggested that Rpf2, Rrs1, rpL5, rpL11, and 5S rRNA might be present in a subcomplex within assembling ribosomes: (1) the pattern of sedimentation of the two assembly factors on sucrose gradients (Fig. 1B, left), and (2) enrichment of Rpf2, Rrs1, rpL5, and rpL11 compared with total proteins from 66S pre-rRNPs when either TAP-tagged Rpf 2 or Rrs 1 was used for affinity purification of complexes from the $\operatorname{rrp1-1}$ mutant (Fig. 1C). When extracts from wild-type and rrp1-1 mutant cells were subjected to centrifugation on $7 \%-47 \%$ sucrose gradients, Rpf2 and Rrs1 were evident in two peaks, one in fractions 15-17 containing 66S preribosomes, and the other in fractions 5-7, where smaller macromolecular complexes $(\sim 450-700 \mathrm{kDa})$ sediment (Fig. 1B, left). Relative to extracts from wild-type strains, mutant extracts contained more Rpf 2 and Rrs 1 in fractions 5-7, and less of each protein in fractions 15-17, suggesting that an extraribosomal subcomplex contain- 
ing Rpf2 and Rrs1 is present in larger amounts in the rrp1-1 mutant strains. Amounts of Rpf2 and Rrs1 in whole-cell extracts remained the same in the rrp1-1 mutant compared with wild-type cells (Fig. 1B, right).

Affinity purification using Rpf2-TAP or Rrs1-TAP from whole-cell extracts of $R R P 1$ and $\operatorname{rrp} 1-1$ strains revealed the proteins normally present in purified $66 \mathrm{~S}$ prerRNPs (Fig. 1C). However, silver staining and Western blotting demonstrated that Rpf2, Rrs1, rpL5, rpL11, rpP0, and Ebp2 were enriched among proteins purified from the rrp1-1 mutant (Fig. 1C,D). These proteins were not enriched when affinity purification was carried out from rrp1-1 mutant extracts using other TAP-tagged ribosome assembly factors such as Nop7, Ssf1, Nug1, or Nsa3 (Supplementary Fig. 1), indicating that these enriched proteins are specifically associated with Rpf2 and Rrs 1 . Immunoblot assays showed that equal amounts of each protein were present in whole-cell extracts of wild-type yeast and the $\operatorname{rrp1-1}$ mutant (Fig. 1D), demonstrating that differential purification did not result simply from the presence of greater amounts of these proteins in the mutant strain. Taken together, these results suggest that Rpf2, Rrs1, rpL5, rpL11, rpP0, and Ebp2 might exist together in a subcomplex.

In order to purify this subcomplex, we fractionated the rrp1-1 mutant extract on sucrose gradients (Fig. 1B, left), then carried out affinity purification from fractions 5-7 using either TAP-tagged Rpf2 or Rrs1. We recovered Rpf2, Rrs1, rpL5, and rpL11 from these fractions (Fig. 1E). In both cases, greater amounts of Rpf 2 and Rrs1 than rpL5 and rpL11 were obtained, suggesting that these fractions might contain a mixture of an Rpf2/Rrs1 heterodimer and the Rpf2/Rrs1/rpL5/rpL11 subcomplex (Rpf2 subcomplex).

RpP0 is located adjacent to 5S rRNA, rpL5, and rpL11 in mature yeast ribosomes (Fig. 1G; Ballesta and Rem- acha 1996). Ebp2 was shown to interact with Rrs1 by a two-hybrid assay (Miyoshi et al. 2002). Thus, it seemed likely that rpP0 and Ebp2 also might be present in the Rpf2 subcomplex. However, these two proteins were not recovered in the purified subcomplex, perhaps due to dissociation during centrifugation or TAP purification.

The discovery that rpL5 and rpL11, two proteins that are adjacent to 5S rRNA in mature ribosomes (Spahn et al. 2001), are present in the Rpf2 subcomplex suggested that the subcomplex might also contain 5S rRNA. To test RNA components of the Rpf2 subcomplex, RNA was extracted from purified subcomplex and hybridized to a microarray that tiles all known yeast noncoding RNAs. Results showed the presence of 5S rRNA in the Rpf2 subcomplex (data not shown). Northern blotting of RNA extracted from the subcomplex confirmed that it does in fact contain $5 \mathrm{~S}$ rRNA, but not $5.8 \mathrm{~S}$ rRNA or $7 \mathrm{~S}$ pre-rRNA (Fig. 1F).

\section{Interactions between proteins in the Rpf2 subcomplex}

In order to map protein-protein interactions within the Rpf2 subcomplex, we performed yeast two-hybrid assays. Pairwise Rpf2-Rrs1, Rpf2-rpL11, and Rrs1-rpL11 interactions in vivo were defined, with interactions between Rpf2 and Rrs1 being stronger than for any other pair of proteins (Supplementary Table 1). To test whether these interactions were direct, GST pull-down assays were carried out in vitro (Fig. 2A). GST-Rpf2 bound specifically to Rrs1. Likewise, GST-Rrs1 interacted directly with Rpf2, and also with rpL11. GSTrpL11 bound to Rpf2. GST-rpL5 bound to Rpf2 and to rpL11, and less well to Rrs1. When tandem affinity purification (TAP) was carried out with extracts treated with a cocktail of phosphatase inhibitors that disrupts pre-rRNPs (Miles et al. 2005), only Rrs1 copurified with
A

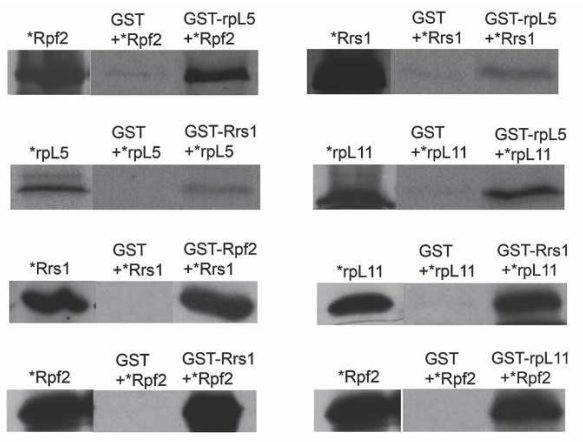

C

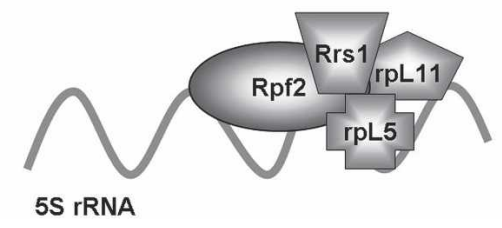

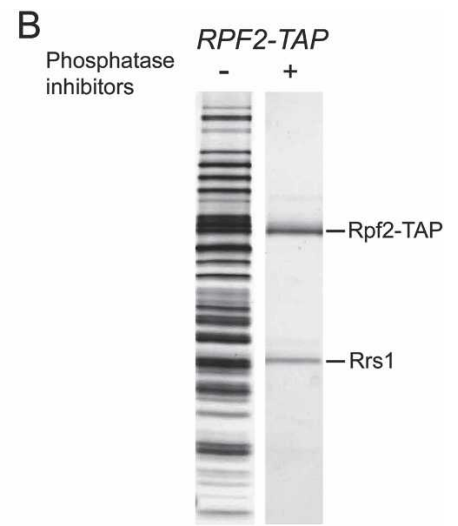

Figure 2. Direct physical interactions between Rpf2, Rrs1, rpL5, and rpL11. (A) Synthetic $\left[{ }^{35} \mathrm{~S}\right]$ methionine-labeled Rpf2, Rrs1, rpL5, or rpL11 proteins $\left(^{*}\right)$ were incubated with GST-Rrs1, Rpf2, rpL5, or rpL11 fusion proteins. (Middle lane) As negative controls, synthetic proteins were incubated with GST beads only. Complexes were eluted from glutathione beads, subjected to SDS-PAGE, and detected by autoradiography. Fifty percent of the input ${ }^{35} \mathrm{~S}$-labeled proteins in each assay (left lane) and $100 \%$ of the pull-down (right lane) are shown. (B) Purification of an Rpf2/Rrs1 heterodimer from whole-cell extracts. Whole-cell extract from wildtype strain JWY7087 was subjected to affinity purification using TAP-tagged Rpf2. Prior to TAP purification, half of the extract was incubated with phosphatase in hibitors, which disrupts pre-rRNPs. Purified proteins were resolved by SDS-PAGE, stained with silver, and identified by mass spectrometry. $(C)$ Protein-protein interactions within the Rpf2 subcomplex. 
TAP-tagged Rpf2 (Fig. 2B), consistent with the strong interactions between Rpf 2 and Rrs 1 observed in the twohybrid and pull-down experiments. These data confirm and extend previous yeast two-hybrid data from Mizuta and coworkers (Miyoshi et al. 2002; Morita et al. 2002; Nariai et al. 2005), leading us to generate a model for protein-protein interactions within the Rpf2 subcomplex (Fig. 2C).

Rpf2 and Rrs1 are required for recruitment of 5S rRNA and ribosomal proteins rpL5 and rpL11 into nascent ribosomes

The presence of 5S rRNA, rpL5, and rpL11 in the Rpf2 subcomplex suggested that assembly factors Rpf2 and Rrs1 might be involved in recruiting these three molecules into preribosomes. Each of the four protein components of the Rpf2 subcomplex is required for production of 60S ribosomal subunits (Moritz et al. 1990; Deshmukh et al. 1993; Morita et al. 2002; Wehner and Baserga 2002). In addition, upon depletion of Rpf 2 or Rrs 1 , processing of $27 \mathrm{SB}$ pre-rRNA is delayed, similar to what occurs when levels of 5S rRNA are diminished (Van Ryk et al. 1992; Dechampesme et al. 1999).

To test whether Rpf2 and Rrs1 are necessary for incorporation of 5S rRNA, rpL5, or rpL11 into nascent ribo- somes, we first assayed the interdependence of these four proteins for assembly into pre-rRNPs. To do so, we affinity-purified preribosomes from strains in which each of the four proteins was individually depleted, using repressible GAL1 promoter fusions of RPF2, RRS1, RPL5, or RPL11. These strains also contained NOP7-TAP to enable purification of $90 \mathrm{~S}$ pre-rRNPs as well as each of the four consecutive 66S pre-rRNPs (Harnpicharnchai et al. 2001). Upon shifting each strain from galactose- to glucose-containing medium, expression of GAL1 promoter constructs was turned off and amounts of each protein subsequently decreased (Supplementary Fig. 2A; Moritz et al. 1990; Deshmukh et al. 1993). Growth of these four strains expressing GAL1 promoter-regulated proteins severely slowed 8-10 h after transfer to glucose, and levels of $60 \mathrm{~S}$ ribosomal subunits specifically decreased (Supplementary Fig. 2B,C; Moritz et al. 1990; Deshmukh et al. 1993).

SDS-PAGE and Western immunoblot analysis of proteins from preribosomal particles purified from each of the four depleted strains demonstrated that pre-rRNPs were largely intact. Most of the preribosomal proteins were recovered in amounts similar to those from unshifted strains (Fig. 3A). Strikingly, amounts of Rpf2, Rrs1, rpL5, and rpL11 were substantially decreased relative to levels of other protein constituents of pre-rRNPs
Figure 3. $5 \mathrm{~S}$ rRNA, rpL5, and $\mathrm{rpL} 11$ are not recruited into 66S preribosomes in the absence of Rpf2 or Rrs1. Yeast strains GAL-RPF2 (JWY8129), GAL-RRS1 (JWY8132), GAL-RPL5 (JWY8108), and GAL-RPL11 (JWY8112) were grown at $30^{\circ} \mathrm{C}$ in galactose medium to $3 \times 10^{7}$ cells per milliliter. A second culture of each strain was grown in galactose medium and shifted to glucose medium for $16 \mathrm{~h}$ to $3 \times 10^{7}$ cells per milliliter to deplete the respective proteins. Whole-cell extracts were subjected to TAP purification, using Nop7-TAP to isolate preribosomes. (A) Proteins present in the affinity-purified preribosomes were resolved by SDSPAGE and stained with silver (left), or subjected to Western blot analysis (right). In each sample pair, proteins from cells grown in galactose-containing medium are on the left and those from cells transferred to glucose are on the right. (B) RNA was extracted from each of the TAP-purified samples described above, as well as from wild-type cells (JWY6938), resolved by denaturing gel electrophoresis, and assayed for 5S rRNA or 27SB pre-rRNA by Northern blotting or primer extension.

A
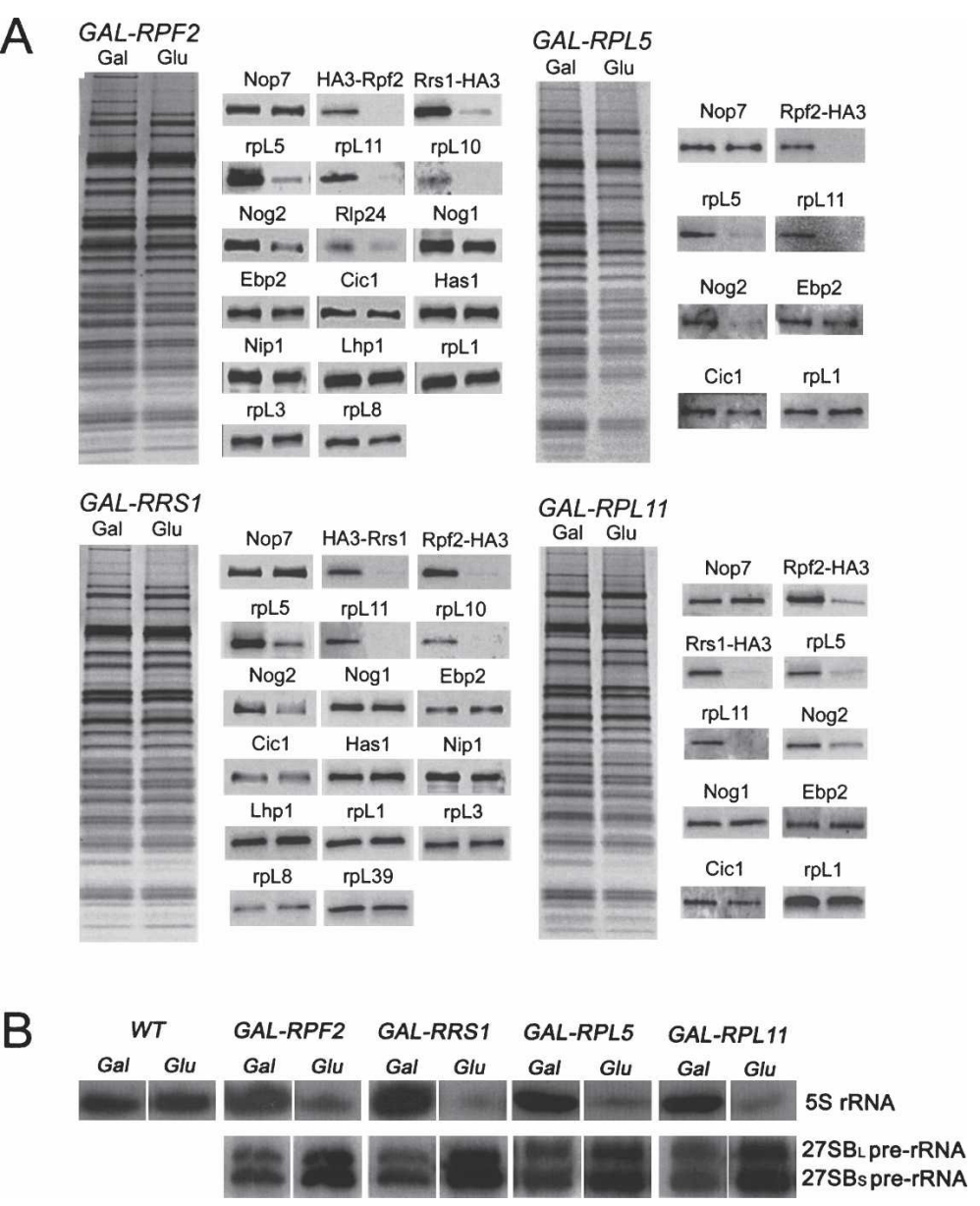
in the four depleted strains. In contrast, when any of 11 other assembly factors was depleted, levels of Rpf2, Rrs1, rpL5, and rpL11 present in preribosomes did not change (data not shown).

Northern blotting demonstrated that amounts of $5 \mathrm{~S}$ rRNA present in $66 \mathrm{~S}$ pre-rRNPs purified from each of the four depleted strains were approximately sevenfold to 12 -fold lower relative to the undepleted strains (Fig. 3B). Amounts of $5 \mathrm{~S}$ rRNA in preribosomes purified from wild-type cells grown in galactose-containing medium or shifted from galactose- to glucose-containing medium were identical, indicating that levels of 5S rRNA in preribosomes are not sensitive to these carbon source changes (Fig. 3B). Consistent with the delay in 27SB prerRNA processing previously observed for Rpf2- and Rrs1depleted strains, preribosomes isolated from the four depleted strains were enriched for those bearing 27SB prerRNA (Fig. 3B). That only modestly larger amounts of 27SB pre-rRNA were recovered may reflect an incomplete block in pre-rRNA processing, together with some turnover of abortive assembly intermediates. Taken together, these results reveal that each of the four proteins in the subcomplex, Rpf2, Rrs1, rpL5, and $\operatorname{rpL11,~is~nec-~}$ essary to recruit all components of the subcomplex, including 5S rRNA, into preribosomal particles.

Interestingly, we also observed a measurable decrease in the amounts of Nog2 and rpL10 present in preribosomes purified from the depleted strains (Fig. 3A). The absence of these two proteins could account in part for the phenotypes observed upon failure to assemble the Rpf2 subcomplex into preribosomes, namely, defects in processing of 27SB pre-rRNA and export of preribosomes from the nucleus (see Discussion).

We independently assayed pre-rRNPs in the Rpf2- and Rrs1-depleted strains using TAP-tagged Ssf1. This assembly factor enters the pathway early, in 90S prerRNPs, dissociates from $66 \mathrm{~S}$ particles containing $27 \mathrm{SB}$ pre-rRNA, and is not present in late $66 \mathrm{~S}$ pre-rRNPs containing 25.5S plus 7S pre-rRNAs (Fatica et al. 2002). Rpf2, Rrs1, rpL5, rpL11, and rpL10 were present in preribosomes purified from wild-type cells using Ssf1-TAP, but were present in much lower amounts when Rpf2 or Rrs1 were depleted (Supplementary Fig. 3). These results confirm our findings with Nop7-TAP, and more importantly, demonstrate that components of the Rpf2 subcomplex associate with preribosomes before Ssf1 dissociates from the pre-rRNPs containing $27 \mathrm{SB}$ pre-rRNA.
Fate of components of the Rpf2 subcomplex when they cannot assemble into preribosomes

What happens to constituents of the Rpf2 subcomplex when they are unable to assemble into preribosomes? Western blotting of whole-cell extracts revealed that both Rpf2 and Rrs1 decreased four- to sevenfold after depletion of any of the other three subcomplex proteins (Fig. 4). Levels of Rrs1 or Rpf2 in cells did not decrease when ribosome assembly was blocked by other means; e.g., in rrp1-1 or nop4-3 mutants (data not shown). While Nog2 was absent from preribosomes upon depletion of each protein in the Rpf 2 subcomplex, levels of it remained the same in whole-cell extracts from the mutants compared with wild-type cells (Fig. 4). Thus, when constituents of the Rpf2 subcomplex cannot associate properly with each other and assemble into preribosomes, Rpf2 and Rrs1 are specifically turned over (Fig. 7, below). In contrast, levels of rpL5 or rpL11 only decreased 1.5-fold to twofold in the four depleted strains (Fig. 4). This smaller decrease in cellular levels of these two ribosomal proteins may simply reflect the fact that the majority of rpL5 and rpL11 present before initiating the depletions is in mature 60S ribosomal subunits and thus is unlikely to turn over upon blocking production of new 605 ribosomes. In the absence of synthesis of new ribosomes, the total number of ribosomes per cell is diminished by dilution upon cell division, which can account in part for the observed decrease in the amounts of rpL5 and rpL11. This is evident from the decreased amounts of $\mathrm{rpL} 1, \mathrm{rpL} 3$, and $\mathrm{rpL} 8$ in whole-cell extracts from the depleted strains (Fig. 4). Nevertheless, a small portion of rpL5 and rpL11 synthesized after depletion of any one of the Rpf2 subcomplex proteins may be unstable. Turnover of unassembled Rpf2 and Rrs1 (Fig. 7, below) may serve as signals to ensure that when $5 \mathrm{~S}$ rRNA, rpL5, and rpL11, three components of mature ribosomes, cannot assemble into preribosomes, biogenesis of 605 subunits does not proceed.

\section{Nuclear export of preribosomes is blocked upon depletion of Rpf2, Rrs1, or rpL5}

Export of preribosomes to the cytoplasm is perturbed in yeast strains expressing lower than wild-type amounts of $5 S$ rRNA, suggesting that proper delivery of 5S rRNA to pre-rRNPs is necessary to produce particles competent for nuclear export (Dechampesme et al. 1999). Thus we

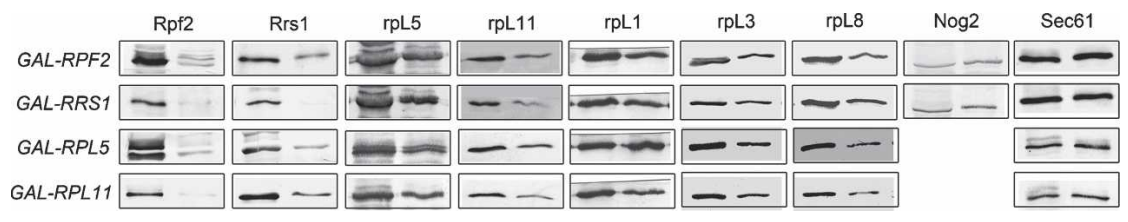
JWY8112) were grown at $30^{\circ} \mathrm{C}$ in galac-
tose-containing medium to $3 \times 10^{7}$ cells per milliliter. A second culture of each grown in galactose medium was shifted to glucose medium for $17 \mathrm{~h}$ to $3 \times 10^{7}$ cells per milliliter. Proteins in whole-cell extracts were assayed by Western blotting. Samples on the left for each pair are from cells grown in galactose medium and those on the right are from cells grown in galactose medium and shifted to glucose medium.

Figure 4. Rpf2 and Rrs1 are unstable upon depletion of each of the Rpf2 subcomplex proteins. Yeast strains GALRPF2 (JWY8129), GAL-RRS1 (JWY8114 or JWY8132), GAL-RPL5 (JWY8108 or JWY8109), and GAL-RPL11 (JWY8080 or JWY8112) were grown at $30^{\circ} \mathrm{C}$ in galac- 
predicted that depletion of any protein in the Rpf 2 subcomplex would block transport of nascent ribosomes from the nucleus to the cytoplasm. To test this idea, we expressed eGFP-tagged rpL25, a reporter for 66S prerRNP export (Hurt et al. 1999), in strains containing GAL1-promoter fusions of RPF2, RRS1, or RPL5. When these strains were shifted from galactose- to glucose-containing medium, eGFP-rpL25 localized primarily in the nucleus (Fig. 5). In contrast, eGFP-rpL25 was located predominantly in the cytoplasm in cells grown in galactose (Figs. 5, 7, below). Previously, Miyoshi et al. (2004) found that nuclear export of preribosomes is blocked in temperature-sensitive rrs 1 mutants. This export defect can be explained by our finding that, similar to the depletion strains, preribosomes purified from the rrs1-84 and rrs1124 mutants lack Rpf2, Rrs1, rpL5, and rpL11 (Supplementary Fig. 4).

\section{Each member of the Rpf2 subcomplex assembles into $90 \mathrm{~S}$ preribosomes}

Identification of pre-rRNAs that copurify with assembly factors enables one to determinewith which of the consecutive assembly intermediates the protein is associated, and thus when each protein assembles into and exits from preribosomes (Harnpicharnchai et al. 2001; Fatica et al. 2002; Saveanu et al. 2003; Miles et al. 2005). To determine when Rpf2 and Rrs1 assemble into prerRNPs, we assayed pre-rRNAs that copurify with Rpf2TAP or Rrs1-TAP. Primer extension results indicated that $35 \mathrm{~S}, 27 \mathrm{SA}_{2}$, and $27 \mathrm{SB}$ pre-rRNAs associate with Rpf2 as well as Rrs1 (Fig. 6A), demonstrating that both
Rpf2 and Rrs1 assemble into 90S pre-rRNPs containing 35S pre-rRNA.

Another way to assay whether assembly factors associate with similar preribosomal particles is to identify the proteins that copurify with those assembly factors. Forty-five different ribosome assembly factors copurify with TAP-tagged Rpf2 or Rrs1 from wild-type cells. All but seven of these proteins were found in both Rpf 2 and Rrs1-containing complexes (Supplementary Table 2), indicating that Rpf2 and Rrs1 are present in similar assembly intermediates.

To determine when rpL5 and $\mathrm{rpL} 11$ are incorporated into preribosomes, we carried out a different set of experiments. Because mature 60S ribosomal subunits are present in great excess compared with preribosomes (>100-fold), it is difficult to specifically assay preribosomal RNAs directly coimmunoprecipitating with ribosomal proteins from whole-cell extracts. To overcome this problem, we first purified 90S and 66S preribosomes away from mature ribosomes using TAP-tagged assembly factor Nop7 (Fig. 6B; Harnpicharnchai et al. 2001). We then assayed with which of these purified pre-rRNPs HA-tagged rpL5 or rpL11 is associated by coimmunoprecipitation using anti-HA antiserum. As observed for Rpf2 and Rrs1, 35S, 27SA 2 , 27SA 3 , and 27SB, pre-rRNAs coprecipitated with both rpL5-HA3 and rpL11-HA3 (Fig. $6 \mathrm{~B})$. Consistent with rpL5 and rpL11 being in early prerRNPs, we could detect these two proteins in particles purified using protein A-tagged early assembly factor Rrp5 (data not shown). Rrp5 is present in 90S preribosomes containing 35S pre-rRNA as well as 66S particles bearing $27 \mathrm{SA}_{2}$ pre-rRNA (de Boer et al. 2006).

\section{Galactose}
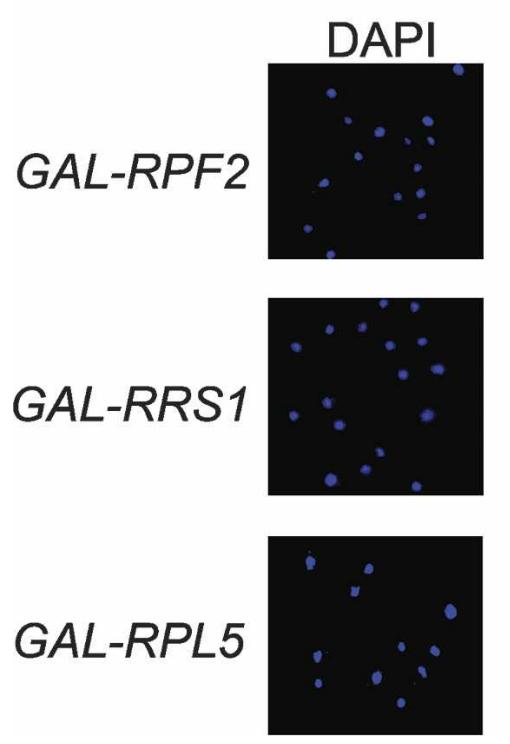
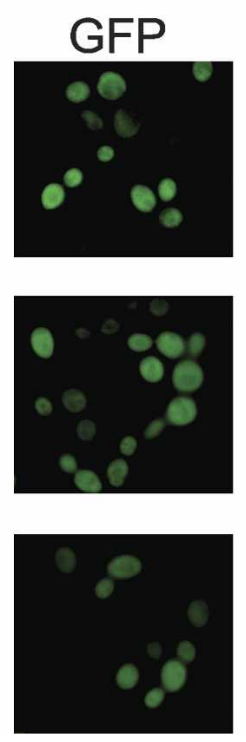

\section{Glucose}
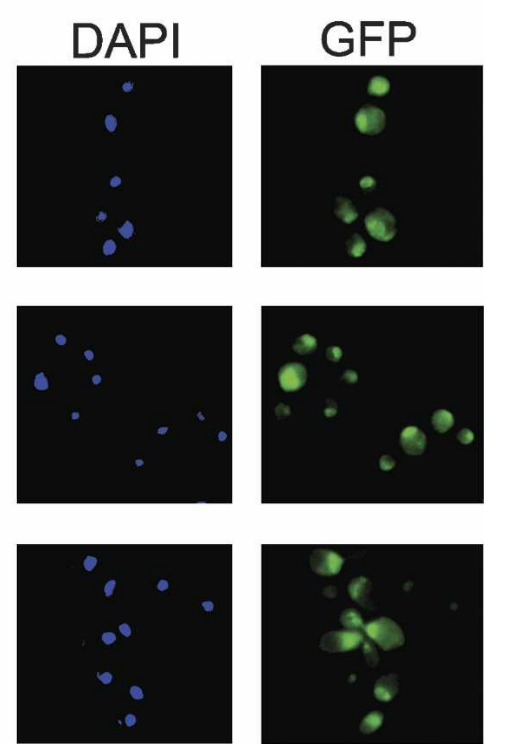

Figure 5. Depletion of Rpf2, Rrs1, or rpL5 causes 66S preribosomes to accumulate in the nucleus. GAL-RPF2 (JWY7703), GAL-RRS1 (JWY7706), or GAL-RPL5 (JWY7696) strains expressing eGFP-tagged rpL25 were grown at $30^{\circ} \mathrm{C}$ in galactose medium to $3 \times 10^{7}$ cells cells per milliliter. A second culture of each strain grown in galactose medium was shifted to glucose medium for $17 \mathrm{~h}$ to $3 \times 10^{7}$ cells per milliliter. Nuclei were stained with DAPI, and ribosomes containing eGFP-rpL25 were detected by fluorescence microscopy. 
A

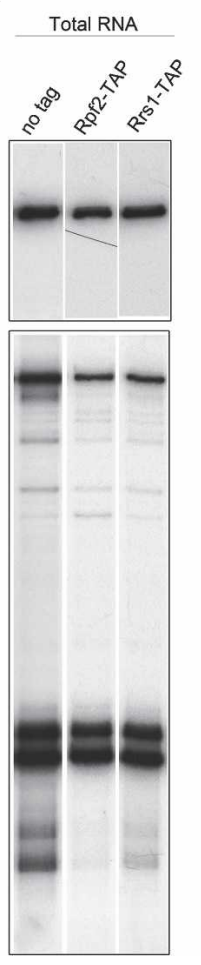

TAP-purified RNA
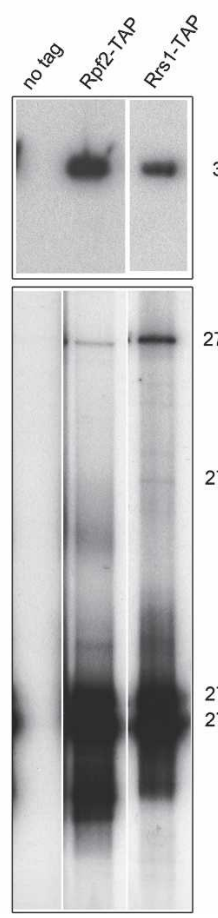

B
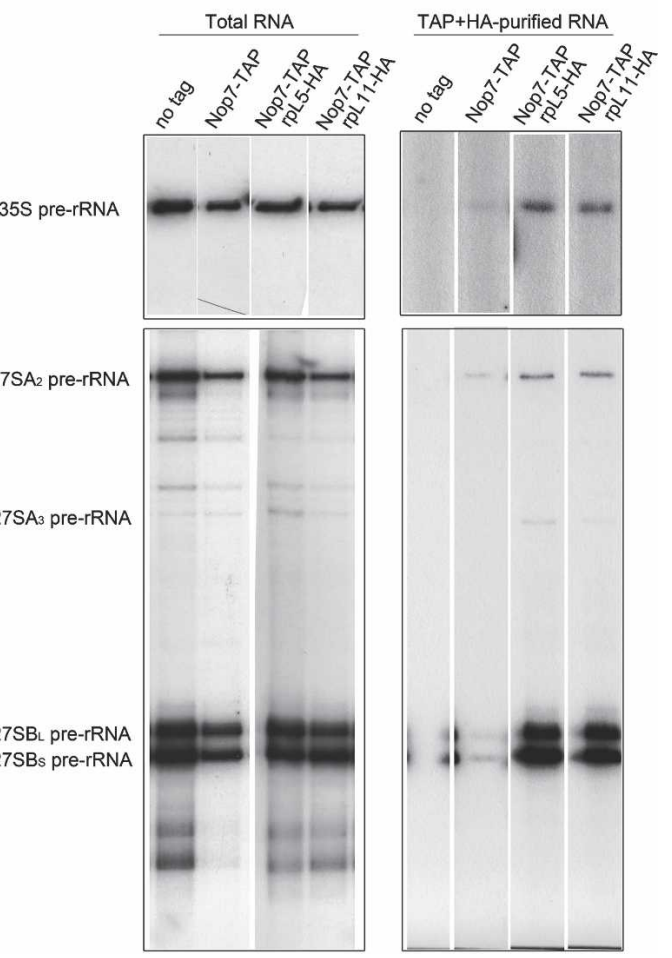

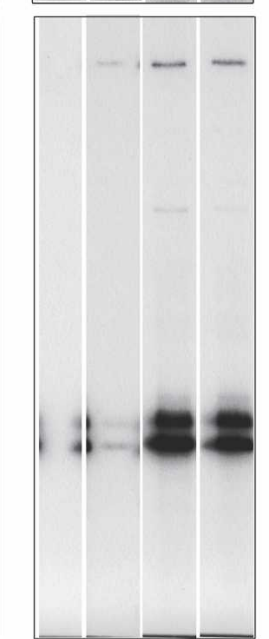

Figure 6. Each component of the Rpf2 subcomplex assembles into 90S preribosomes. (A) Whole-cell extracts were prepared from an untagged strain (JWY6147) and Rpf2-TAP (JWY7087) and Rrs1-TAP (JWY7461) strains. RNA was extracted from whole-cell extracts (left) and affinity-purified samples (right). Five micrograms of total RNA and $100 \%$ of purified RNA were used to assay $35 \mathrm{~S}, 27 \mathrm{SA}_{2}$, and $27 \mathrm{SB}$ pre-rRNAs by primer extension. Amounts of $27 \mathrm{SA}_{3}$ pre-rRNA were very low and thus are invisible in some lanes. No prerRNAs copurified upon mock purification from untagged strains. Note that different oligos were used in reactions for $35 \mathrm{~S}$ and $27 \mathrm{~S}$ pre-rRNAs, and longer exposure was done for $35 \mathrm{~S}$ pre-rRNA. $(B)$ Preribosomes were affinitypurified using TAP-tagged Nop7. Purified prerRNPs containing rpL5-HA3 or rpL11 were immunoprecipitated by anti-HA antiserum. Five micrograms of total RNA (left) and 100\% of purified RNA (right) were assayed for 35S, $27 \mathrm{SA}_{2}, 27 \mathrm{SA}_{3}$, and $27 \mathrm{SB}$ pre-rRNAs by primer extension. Pre-rRNPs from an untagged strain were used as a negative control.
Previously it was observed that rpL5 binds to 5S rRNA soon after it is synthesized and accompanies 5S rRNA into ribosomes (Steitz et al. 1988). Yeast 5S rRNA was found in an extraribosomal complex with rpL5, indicating that yeast rpL5 also associates with 5S rRNA before incorporation into preribosomes (Deshmukh et al. 1993). Taken together, these results establish that Rpf2, Rrs1, rpL5, rpL11, and 5S rRNA assemble into 90S preribosomes containing 35S pre-rRNA (Fig. 7). Intriguingly, this point of entry is well before the first detectable requirement of these molecules for ribosome biogenesis, namely, processing of $27 \mathrm{SB}$ pre-rRNA (see Discussion).

\section{More detailed characterization of preribosomes containing Rpf2}

Recent technological advances have made it possible to affinity purify preribosomal complexes more rapidly by using a single-step isolation method (Oeffinger et al. 2007); this method allows us to capture transiently interacting components, giving us a more comprehensive picture of preribosomal particle composition. In order to better define Rpf2-containing preribosomes, we applied the single-step purification method to protein A-tagged Rpf2. Copurifying proteins were resolved by SDS-PAGE, visualized by Coomassie staining, and gels were sliced into 1-mm sections. MS and MS/MS were used to identify proteins in each slice (Krutchinsky et al. 2001). Using this approach, we identified 51 different ribosome assembly factors copurifying with Rpf2-ProtA compared with 43 factors using TAP-tagged Rpf2 (Supplementary
Table 2). These additional proteins included several early and late $60 \mathrm{~S}$ components that are most likely to be present in lower amounts than most other assembly factors in the total population of Rpf2-associated 90S and 66S pre-rRNPs. Moreover, we were able to coisolate the exonucleases Rat1 and Xrn1-as well as the cofactor for Rat1, Rai1-with preribosomes for the first time, by affinity purification, which had not been possible using TAP (Johnson 1997; Xue et al. 2000).

\section{Discussion}

Purifying and characterizing neighborhoods of molecules within assembling ribosomes have enabled us to investigate in more detail the structure of assembling ribosomes, the functions of ribosome assembly factors, and the pathway of ribosome assembly. We identified and purified a ribonucleoprotein complex containing yeast ribosome assembly factors Rpf2 and Rrs1, ribosomal proteins rpL5 and rpL11, and 5S rRNA. Components of this subcomplex join pre-rRNPs at an early step in the assembly pathway, forming a stable neighborhood within $90 \mathrm{~S}$ preribosomes. However, the functions in ribosome biogenesis of molecules in this neighborhood are only evident at later maturation steps, processing of $27 \mathrm{SB}$ prerRNA and proper release of pre-rRNPs from the nucleolus. Upon depletion of each of these four proteins, the other three proteins and 5S rRNA fail to be recruited into pre-rRNPs. Preribosomes lacking the constituents of the Rpf2 neighborhood are largely intact, but they cannot undergo further maturation, are prematurely released 


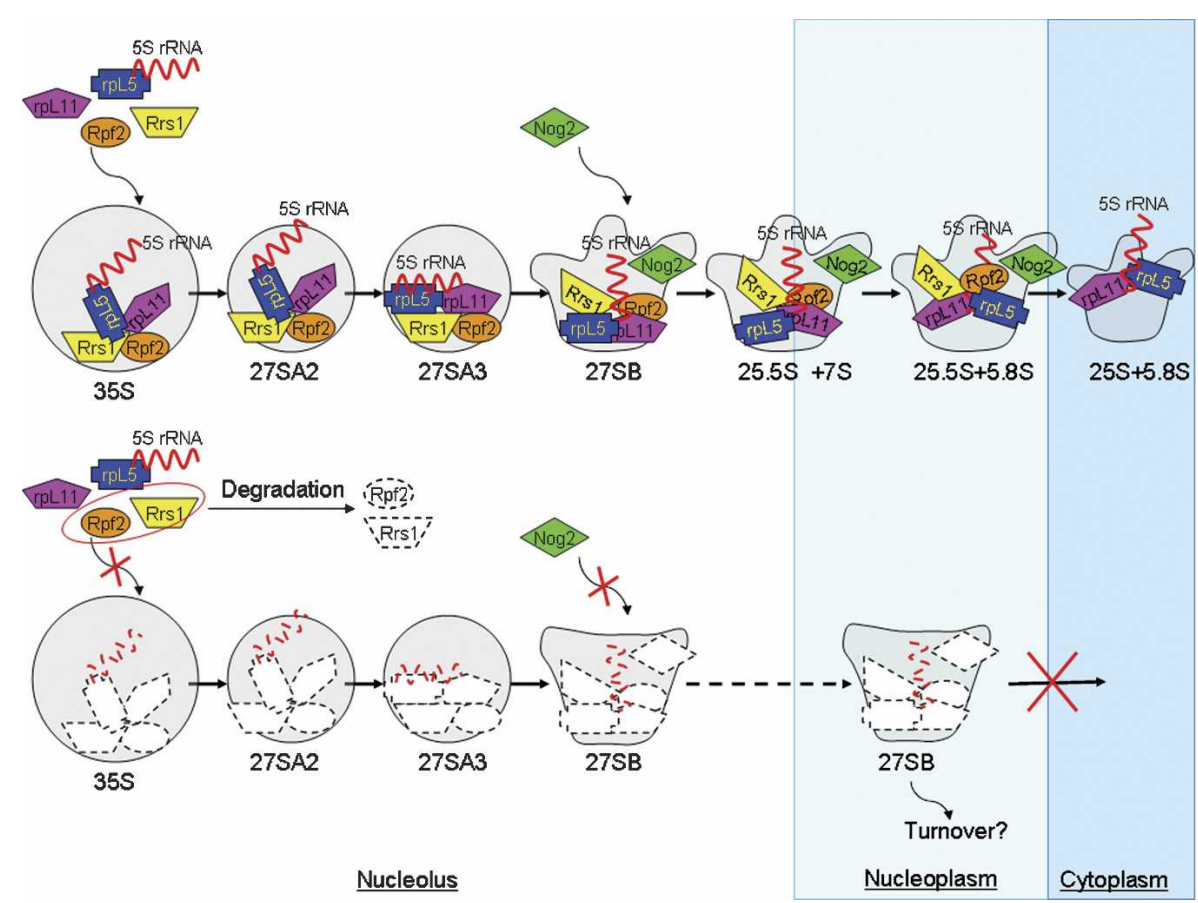

Figure 7. Model for recruitment and function of 5S rRNA, RpL5, and RpL11. (Top) In wild-type cells, Rpf2 and Rrs1 help to recruit rpL5, rpL11, and 5S rRNA into 90S preribosomal particles containing 35S pre-rRNA. (Bottom) When components of the subcomplex cannot assemble into $90 \mathrm{~S}$ particles, $35 \mathrm{~S}, 27 \mathrm{SA}_{2}, 27 \mathrm{SA}_{3}$ pre-rRNAs can be processed efficiently. However, processing of $27 \mathrm{SB}$ pre-rRNA is blocked. The abortive pre-rRNPs are released from the nucleolus to the nucleoplasm, and cannot be exported to the cytoplasm. When the subcomplex cannot be assembled, Rpf2 and Rrs1 are unstable.

from the nucleolus to the nucleoplasm, and cannot be exported to the cytoplasm.

\section{Discovering neighborhoods in assembling ribosomes}

Discovery of the Rpf2 subcomplex (Fig. 1) and characterization of direct interactions among the protein components (Fig. 2) support and extend previous observations: (1) Yeast rpL5 and rpL11 and their bacterial homologs L18 and L5 are adjacent to 5S rRNA in the central protuberance of mature 60S (or 50S) ribosomal subunits (Tsay et al. 1994; Spahn et al. 2001; Klein et al. 2004), and (2) two-hybrid assays identified pairwise interactions among Rpf2, Rrs1, rpL5, and rpL11 (Miyoshi et al. 2002; Morita et al. 2002; Nariai et al. 2005).

It is becoming evident that assembly factors, in some cases together with RNA molecules, may form small complexes either prior to entering preribosomal particles or within nascent ribosomes (Harnpicharnchai et al. 2001; Dosil and Bustelo 2004; Krogan et al. 2004; Nissan et al. 2004; Miles et al. 2005; Lebreton et al. 2006; Rosado et al. 2007). Some of these neighborhoods are evident in wild-type cells. However, others are not, perhaps because they only assemble and exist within preribosomes. Alternatively, those that do form before entering prerRNPs may very quickly assemble into nascent ribosomes, such that amounts of free subcomplexes are not readily detectable in extracts from wild-type cells.

In order to identify neighborhoods that are not evident in large amounts in wild-type cells, such as the Rpf2 subcomplex, we used the rrp1-1 mutant, in which biogenesis of $60 \mathrm{~S}$ ribosomal subunits is blocked and abortive $66 \mathrm{~S}$ assembly intermediates fall apart into subcomplexes (Harnpicharnchai et al. 2001; Horsey et al. 2004; Miles et al. 2005). Dissociation of 665 pre-rRNPs into smaller particles may result in part from degradation of the $27 \mathrm{~S}$ pre-rRNAs within them, mediated by a nuclear surveillance and degradation pathway; e.g., the TRAMP complex and the exosome (Dez et al. 2006). Occasional errors in ribosome assembly in wild-type cells, also resulting in degradation of some preribosomes (Allmang et al. 2000; Kuai et al. 2004), could account for the small amount of Rpf2 and Rrs1 in wild-type extracts that sediments as an $\sim 450$ - to $700-k D a$ complex (Fig. 1B). The Rpf2 subcomplex assembles into 90S particles, which is upstream of the block in processing of $27 \mathrm{SA}_{3}$ pre-rRNA in the rrp1-1 mutant. Therefore, we believe that most, if not all, of the Rpf2 subcomplex that we purified from the rrp1-1 mutant had already assembled into preribosomes but dissociated from the abortive 66S $\mathrm{A}_{3}$ particles. The pattern of physical and functional interactions and the mutual interdependence among Rpf2, Rrs1, rpL5, rpL11, and 5S rRNA convince us that the Rpf2 subcomplex is a specific ribosome assembly intermediate rather than an artifact of the rrp1-1 mutant. A combination of different approaches (Harnpicharnchai et al. 2001; Dosil and Bustelo 2004; Krogan et al. 2004; Nissan et al. 2004; Miles et al. 2005; Lebreton et al. 2006; this study) should facili- 
tate identification of additional neighborhoods, enabling better characterization of physical and functional interactions between preribosomal molecules, and eventually helping define the global structure of consecutive preribosomal particles.

The Rpf2 subcomplex functions in intermediate stages of 665 preribosome maturation and is necessary to recruit assembly factor Nog2 and ribosomal protein rpL10

Processing of 27SB pre-rRNA and subsequent nuclear export of preribosomes are thought to involve complex rearrangements of higher-order structures of pre-rRNAs and pre-rRNPs (Côté et al. 2002), and thus may require the concerted action of a number of assembly factors and ribosomal proteins. Our results indicate that incorporation of 5S rRNA, rpL5, and rpL11 into preribosomes, mediated by Rpf 2 and Rrs1, may help trigger these late steps in maturation of 66S pre-rRNPs (Fig. 7). Previously, it was found that depletion or inactivation of 5S rRNA, Rpf2, or Rrs1 delays processing of 27SB pre-rRNA and blocks nuclear export of 66S particles (Dechampesme et al. 1999; Morita et al. 2002; Wehner and Baserga 2002; Miyoshi et al. 2004). Recently, Yao et al. (2007) found that Mex67 and Mtr2 are necessary for nuclear export of 66S preribosomes and bind to 5S rRNA. Our work explains these phenotypes and ties them together by showing that Rpf2, Rrs1, rpL5, and rpL11 are present together in the Rpf2 subcomplex, and are necessary for recruiting each other, as well as 5S rRNA, into nascent ribosomes (Figs. 3, 7).

Upon depletion of the Rpf2 subcomplex proteins, assembly factor Nog2 is absent from preribosomes. Nog2 is a putative GTPase required for processing of $27 \mathrm{SB}$ prerRNA (Saveanu et al. 2001). Thus, the defect in 27SB pre-rRNA processing observed upon depletion of each of the Rpf2 subcomplex proteins may be partially due to the absence of Nog2 (Fig. 7). As a GTPase, Nog2 may rearrange $66 \mathrm{~S}$ pre-rRNP structure to create the proper conformation required for further maturation steps, including processing of the spacer sequence ITS2 and nuclear export of preribosomes.

We also found that depletion of Rpf2 subcomplex proteins prevents stable association of $\mathrm{rpL} 10$ with preribosomes (Fig. 3A). RpL10 is required for nuclear export of nascent 60S subunits (Gadal et al. 2001). Thus, the observed defects in nuclear export when the Rpf2 subcomplex does not assemble into preribosomes may result in part from the absence of rpL10 from pre-rRNPs. Although previous results suggested that $\mathrm{rpL10}$ does not associate with pre-rRNPs until they reach the cytoplasm (West et al. 2005), we found rpL10 present in early preribosomal particles. As shown in Supplementary Figure 3, when early assembly factor Ssf1 was TAP-tagged to purify preribosomes, rpL10 was identifed in pre-rRNPs purified from wild-type cells. However, rpL10 was present in much lower amounts in Ssf1-containing particles when Rpf2 or Rrs1 was depleted. To reconcile these discrepancies, we propose that, analogous to acidic ribo- somal protein interactions with ribosomes, rpL10 cycles on and off preribosomes (Zinker and Warner 1976; Ballesta and Remacha 1996). Since rpL5, 5S rRNA, and rpL11 lie adjacent to rpL10 in mature ribosomes (Fig. 1G), their absence from pre-rRNPs may create local distortions in the particles, which might prevent rpL10 from assembling into early pre-rRNPs or rejoining later pre-rRNPs. Consistent with this hypothesis, we found that preribosomal particles are intact upon depletion of rpL10 (data not shown), indicating that the absence of rpL10 from pre-rRNPs can be tolerated.

\section{S rRNA, rpL5, and rpL11 assemble into nascent ribosomes at an early step}

To understand the functions of assembly factors and ribosomal proteins, it is useful to determine when each assembly factor and ribosomal protein assembles into preribosomes. Based on the results that the consecutive pre-rRNA processing intermediates $35 \mathrm{~S}, 27 \mathrm{SA}_{2}, 27 \mathrm{SA}_{3}$, and $27 \mathrm{SB}$ pre-rRNAs copurify with each of the four proteins in the Rpf2 subcomplex (Fig. 6), we believe that each component of the neighborhood assembles into $90 \mathrm{~S}$ preribosomes containing 35 S pre-rRNA (Fig. 7). That different amounts of 35S pre-rRNA copurify with each of the four proteins might reflect at least two possible assembly mechanisms: (1) Rpf2, Rrs1, rpL5/5S rRNA, and rpL11 might assemble into 90S particles at distinct consecutive substeps. Thus, these five molecules might form a stable neighborhood only after their incorporation into nascent ribosomes. (2) Alternatively, the complete Rpf2 subcomplex might form before joining 90S particles. Using different epitope tags or purification approaches may cause different amounts of pre-rRNAs to copurify with each protein component of the subcomplex. Although these technical limitations prevent us from distinguishing whether components of the Rpf2 subcomplex enter $90 \mathrm{~S}$ particles before or after assembling with each other, our coimmunoprecipitation experiments do indicate when rpL5/5S rRNA and rpL11 assemble into preribosomes and provide a general approach to accurately map entry points of ribosomal proteins.

Rpf2 contains an RNA-binding motif essential for its function (Wehner and Baserga 2002), and thus may play a key role in targeting 5S rRNA, rpL5, and rpL11 into $90 \mathrm{~S}$ pre-rRNPs. GST pull-down data in Figure 2A show direct interactions between Rpf 2 and rpL5 or rpL11. In archaebacteria, 5S rRNA is anchored in ribosomes by contacts with five ribosomal proteins and by direct interactions with 23S rRNA (Klein et al. 2004). Thus, stable insertion of 5S rRNA into yeast pre-rRNPs may require establishment of one or more sequential protein-RNA and RNARNA interactions. Rpf2 might target rpL5/5S rRNA and rpL11 into 90S particles through interactions with 5S rRNA or 35S pre-rRNA, as well as through protein-protein interactions.

Rpf2, Rrs1, rpL5, rpL11, and 5S rRNA assemble into the 90S particle, but are only necessary for a later step in the assembly pathway, processing of $27 \mathrm{SB}$ pre-rRNA. 
Thus, in the absence of the Rpf2 subcomplex, preribosomes can still proceed through several early maturation steps to efficiently process $35 \mathrm{~S}, 27 \mathrm{SA} 2$, and $27 \mathrm{SA} 3$ prerRNAs (Fig. 7). The apparent lack of dependence on the Rpf2 subcomplex of these early steps in ribosome maturation might be explained as follows. Components of the subcomplex may function as scaffolding molecules to recruit ribosomal proteins or other assembly factors that only function later, such as Nog2. Alternatively, the Rpf2 subcomplex molecules may help establish and maintain local RNP architecture required in later steps in subunit biogenesis. Such a local distortion caused by the absence of the Rpf2 subcomplex would not be evident by our current assays.

\section{Materials and methods}

Yeast strains (Supplementary Table 3) were grown and harvested as described in Horsey et al. (2004). 3HA- and TAP-tagged genes and GAL1 promoter fusions were generated as described in Longtine et al. (1998) and Harnpicharnchai et al. (2001). Sequences of oligonucleotides used for PCR to tag genes are available on request.

Subcomplexes, preribosomes, ribosomes, and polyribosomes were resolved on $7 \%-47 \%$ sucrose gradients as described in Harnpicharnchai et al. (2001). TAPs of preribosomes or subcomplexes from whole-cell extracts or subcellular fractions were carried out by the methods of Miles et al. (2005). One-step purification of preribosomes from whole-cell extracts was performed as described (Oeffinger et al. 2007). RNAs from wholecell extracts or enriched by TAP purification were assayed by Northern analysis or primer extension (Horsey et al. 2004). Proteins present in whole-cell extracts, sucrose gradient fractions, purified preribosomes, or assembly subcomplexes were assayed by Western blot analysis (Ausubel et al. 1994). TAP-tagged proteins were detected using alkaline phosphatase conjugated to IgG (Pierce). 3HA-tagged proteins were identified with mouse monoclonal antibody 12CA5. Rabbit polyclonal or mouse monoclonal antibodies were used against ribosomal or nonribosomal proteins.

Proteins copurifying with TAP-tagged Rpf 2 or Rrs 1 or protein A-tagged Rpf2 were identified by mass spectrometry as described in Eng et al. (1994), Horsey et al. (2004), Guo et al. (2005), and Krutchinsky et al. (2001).

Two-hybrid and GST pull-down assays of protein-protein interactions were performed as described in Miles et al. (2005). Release of preribosomes from nucleoli and export from the nucleoplasm to the cytoplasm were assayed using the rpL25-eGFP reporter system, as described in Hurt et al. (1999), using a Zeiss Axioskop 2 plus microscope.

\section{Acknowledgments}

We thank Keiko Mizuta (Hiroshima University) for generously providing strains KM129 and KM403. We thank Mike Snyder (Yale University) for yeast strains expressing GST fusion proteins, Cosmin Saveanu for GST-RPL5, and Stan Fields and Tony Hazbun (University of Washington) for two-hybrid plasmids. We are grateful to the following people for generously providing antibodies: John Aris (Nop2), Juan Pedro Ballesta (rpP0), Jesus de la Cruz and Patrick Linder (Has 1), David Goldfarb (Nip7), Janine Maddock and Jennifer Fuentes (Nog1), Michael McAlear (Ebp2), Cosmin Saveanu (rpL24, Nog2), Maurice Swanson (rpL39), Bernard Trumpower (rpL10), Arlen Johnson (rpL8), Jon Warner (rpL3), Francois Lacroute (rpL1), Elisabeth Tosta (Cic1), Sandra
Wolin (Lhp1), and Jeff Brodsky (Sec61). We thank Susan Dowd, Mark Bier, Karen Wei, Jeffrey DeGrasse, and Brian Chait for assistance with mass spectrometry. We thank Jonathan Dinman, Arlen Johnson, and Jon Warner, as well as members of our laboratory, for fruitful discussions and for comments on the manuscript. We thank Brooke McCartney for use of her microscope. This work was supported by NIH grant GM28301 to J.L.W., NHLBI grant N01-HV-28180 to Y.G., funds from the government of Thailand to P.H., NIH grant NCRR 5 U54 RR022220 to M.O. and M.P.R., a CIHR fellowship to S.H., and a grant from CIHR to T.R.H. (MOP-49451).

\section{References}

Allmang, C., Mitchell, P., Petfalski, E., and Tollervey, D. 2000. Degradation of ribosomal RNA precursors by the exosome. Nucleic Acids Res. 28: 1684-1691.

Andrew, C., Hopper, A.K., and Hall, B.D. 1976. A yeast mutant defective in the processing of $27 \mathrm{~S}$ r-RNA precursor. Mol. Gen. Genet. 144: 29-37.

Ausubel, F.M., Brent, R., Kingston, R.E., Moore, D.D., Seidman, J.G., Smith, J.A., and Struhl, K. 1994. Current protocols in molecular biology. John Wiley \& Sons, Inc., New York

Ballesta, J.P. and Remacha, M. 1996. The large ribosomal subunit stalk as a regulatory element of the eukaryotic translational machinery. Prog. Nucleic Acids Res. Mol. Biol. 55: 157-193.

Côté, C.A., Greer, C.L., and Peculis, B.A. 2002. Dynamic conformational model for the role of ITS2 in pre-rRNA processing in yeast. RNA 8: 786-797.

de Boer, P., Vos, H.R., Faber, A.W., Vos, J.C., and Raué, H.A. 2006. Rrp5p, a trans-acting factor in yeast ribosome biogenesis, is an RNA-binding protein with a pronounced preference for U-rich sequences. RNA 12: 263-271.

Dechampesme, A.M., Koroleva, O., Leger-Silverstre, I., Gas, N., and Camier, S. 1999. Assembly of 5S ribosomal RNA is required at a specific step of the pre-rRNA processing pathway. J. Cell Biol. 145: 1369-1380.

Deshmukh, M., Tsay, Y.F., Paulovich, A.G., and Woolford Jr., J.L. 1993. Yeast ribosomal protein L1 is required for the stability of newly synthesized 5S rRNA and the assembly of $60 \mathrm{~S}$ ribosomal subunits. Mol. Cell. Biol. 13: 2835-2845.

Dez, C., Houseley, J., and Tollervey, D. 2006. Surveillance of nuclear-restricted pre-ribosomes within a subnucleolar region of Saccharomyces cerevisiae. EMBO J. 25: 1534-1546.

Dosil, M. and Bustelo, X.R. 2004. Functional characterization of Pwp2, a WD family protein essential for the assembly of the 90 S pre-ribosomal particle. I. Biol. Chem. 279: 37385-37397.

Eisinger, D.P., Dick, F.A., Denke, E., and Trumpower, B.L. 1997. SQT1, which encodes an essential WD domain protein of Saccharomyces cerevisiae, suppresses dominant-negative mutations of the ribosomal protein gene QSR1. Mol. Cell. Biol. 17: 5146-5155.

Eng, J.K., McCormack, A.L., and Yates III, J.R. 1994. An approach to correlate tandem mass spectral data of peptides with amino acid sequences in a protein database. I. Am. Mass Spectrom. 5: 976-989.

Fatica, A. and Tollervey, D. 2002. Making ribosomes. Curr. Opin. Cell Biol. 14: 313-318.

Fatica, A., Cronshaw, A.D., Dlakic, M., and Tollervey, D. 2002. Ssf1p prevents premature processing of an early pre-60 ribosomal particles. Mol. Cell 9: 341-351.

Ferreira-Cerca, S., Pöll, G., Gleizes, P.E., Tschochner, H., and Milkereit, P. 2005. Roles of eukaryotic ribosomal proteins in 
maturation and transport of pre-18S rRNA and ribosome function. Mol. Cell 20: 263-275.

Fromont-Racine, M., Senger, B., Saveanu, C., and Fasiolo, F. 2003. Ribosome assembly in eukaryotes. Gene 313: 17-42.

Gadal, O., Strauss, D., Kessl, J., Trumpower, B., Tollervey, D., and Hurt, E. 2001. Nuclear export of 60 S ribosomal subunits depends on Xpolp and requires a nuclear export sequencecontaining factor, $\mathrm{Nmd} 3 \mathrm{p}$, that associates with the large subunit protein Rpl10p. Mol. Cell. Biol. 21: 3405-3415.

Granneman, S. and Baserga, S.J. 2004. Ribosome biogenesis: Of knobs and RNA processing. Exp. Cell Res. 296: 43-50.

Gray, P.N., Garrett, R.A., Stoffler, G., and Monier, R. 1972. An attempt at the identification of the proteins involved in the incorporation of 5S RNA during 50S ribosomal subunit assembly. Eur. J. Biochem. 28: 412-421.

Guddat, W., Bakken, A.H., and Pieler, T. 1990. Protein mediated nuclear export of RNA: rRNA containing small RNPs in Xenopus oocytes. Cell 60: 619-628.

Guo, Y., Ma, S., Grigoryev, D., Van Eyk, J., and Garcia, J.G.N. 2005. 1-DE MS and 2-D LC-MS analysis of the mouse bronchoalveolar lavage proteome. Proteomics 5: 4608-4624.

Harnpicharnchai, P., Jakovljevic, J., Horsey, E., Miles, T., Roman, J., Rout, M., Meagher, D., Imai, B., Guo, Y., Brame, C.J., et al. 2001. Composition and functional characterization of yeast 66S ribosome assembly intermediates. Mol. Cell 8: 505-515.

Held, W.A., Mizushima, S., and Nomura, M. 1973. Reconstitution of Escherichia coli 30S ribosomal subunits from purified molecular components. J. Biol. Chem. 248: 5720-5730.

Horsey, E.W., Jakovljevic, J., Miles, T.D., Harnpicharnchai, P., and Woolford Jr., J.L. 2004. Role of the yeast Rrp1 protein in the dynamics of pre-ribosome maturation. RNA 10: 813827.

Hurt, E., Hannus, S., Schmeizl, B., Lau, D., Tollervey, D., and Simos, G. 1999. A novel in vivo assay reveals inhibition of ribosomal nuclear export in Ran-cycle and nucleoporin mutants. J. Cell Biol. 144: 389-401.

Iouk, T.L., Aitchison, J.D., Maguire, S., and Wozniak, R.W. 2001. Rrb1p, a yeast nuclear WD-repeat protein involved in the regulation of ribosome biosynthesis. Mol. Cell. Biol. 21: 1260-1271.

Johnson, A.W. 1997. Ratlp and Xrnlp are functionally interchangeable exoribonucleases that are restricted to and required in the nucleus and cytoplasm, respectively. Mol. Cell. Biol. 17: 6122-6130.

Kiparisov, S., Petrov, A., Meskauskas, A., Sergiev, P.V., Dontsova, O.A., and Dinman, J.D. 2005. Structural and functional analysis of 5S rRNA in Saccharomyces cerevisiae. Mol. Genet. Genomics 274: 235-247.

Klein, D.J., Moore, P.B., and Steitz, T.A. 2004. The roles of ribosomal proteins in the structure assembly, and evolution of the large ribosomal subunit. J. Mol. Biol. 340: 141-177.

Krogan, N., Peng, W.-T., Cagney, G., Robinson, M.D., Haw, R., Zhong, G., Guo, X., Zhang, X., Canadien, V., Richards, D.P., et al. 2004. High-definition macromolecular composition of yeast RNA-processing complexes. Mol. Cell 13: 225-239.

Kruiswijk, T., Planta, R.J., and Krop, J.M. 1978. The course of the assembly of ribosomal subunits in yeast. Biochim. Biophys. Acta 517: 378-389.

Krutchinsky, A.N., Kalkum, M., and Chait, B.T. 2001. Automatic identification of proteins with a MALDI-quadrupole ion trap mass spectrometer. Anal. Chem. 73: 5066-5077.

Kuai, L., Fang, F., Butler, J.S., and Sherman, F. 2004. Polyadenylation of rRNA in Saccharomyces cerevisiae. Proc. Natl. Acad. Sci. 101: 8581-8586.

Lebreton, A., Saveanu, C., Decourty, L., Rain, J.C., Jacquier, A., and Fromont-Racine, M. 2006. A functional network involved in the recycling of nucleocytoplasmic pre-60S factors. J. Cell Biol. 173: 349-360.

Longtine, M.S., McKenzie III, A., DeMarini, D.J., Shah, N.G., Wach, A., Brachat, A., Philippsen, P., and Pringle, J.R. 1998. Additional modules for versatile and economical PCR-based gene deletion and modification in Saccharomyces cerevisiae. Yeast 14: 953-961.

Miles, T.D., Jakovljevic, J., Horsey, E.W., Harnpicharnchai, P., Tang, L., and Woolford Jr., J.L. 2005. Ytm1, Nop7, and Erb1 form a complex necessary for maturation of yeast $66 \mathrm{~S}$ preribosomes. Mol. Cell. Biol. 25: 10419-10432.

Miyoshi, K., Tsujii, R., Yoshida, H., Maki, Y., Wada, A., Matsui, Y., Toh-E, A., and Mizuta, K. 2002. Normal assembly of $60 \mathrm{~S}$ ribosomal subunits is required for the signaling in response to a secretory defect in Saccharomyces cerevisiae. J. Biol. Chem. 277: 18334-18339.

Miyoshi, K., Shirai, C., Horigome, C., Takenami, K., Kawasaki, J., and Mizuta, K. 2004. Rrs1p, a ribosomal protein L11-binding protein, is required for nuclear export of the $60 \mathrm{~S}$ preribosomal subunit in Saccharomyces cerevisiae. FEBS Lett. 565: 106-110.

Morita, D., Miyoshi, K., Matsui, Y., Toh, E.A., Shinkawa, H., Miyakawa, T., and Mizuta, K. 2002. Rpf2p, an evolutionarily conserved protein, interacts with ribosomal protein L11 and is essential for the processing of $27 \mathrm{SB}$ pre-rRNP to $25 \mathrm{~S}$ rRNA and the 60S ribosomal subunit assembly in Saccharomyces cerevisiae. J. Biol. Chem. 277: 28780-28786.

Moritz, M., Paulovich, A.G., Tsay, Y.F., and Woolford Jr., J.L. 1990. Depletion of yeast ribosomal proteins L16 or rp59 disrupts ribosome assembly. J. Cell Biol. 111: 2261-2274.

Nariai, M., Tanaka, T., Okada, T., Shirai, C., Horigome, C., and Mizuta, K. 2005. Synergistic defect in 60 S ribosomal subunit assembly caused by a mutation of Rrs $1 \mathrm{p}$, a ribosomal protein L11-binding protein, and 3'-extension of 5S rRNA in Saccharomyces cerevisiae. Nucleic Acids Res. 33: 4553-4562.

Nissan, T.A., Galani, K., Maco, B., Tollervey, D., Aebi, U., and Hurt, E. 2004. A pre-ribosome with a tadpole-like structure functions in ATP-dependent maturation of $60 \mathrm{~S}$ subunits. Mol. Cell 15: 295-301.

Oeffinger, M., Wei, K.E., Rogers, R., DeGrasse, J.A., Chait, B.T., John, D., Aitchison, J.D., and Rout, M.P. 2007. Comprehensive analysis of diverse ribonucleoprotein complexes. Nat. Methods (in press).

Picard, J. and Wegnez, M. 1979. Isolation of a 7S particle from Xenopus laevis oocytes: A 5S RNA-protein complex. Proc. Natl. Acad. Sci. 76: 241-245.

Raué, H.A. 2003. Pre-ribosomal RNA processing and assembly in Saccharomyces cerevisiae: The machine that makes the machine. In The nucleolus (ed. M.O.J. Olson), pp. 1-24. Kulwer Academic/Plenum Publishers, New York, NY.

Rinke, J. and Steitz, J.A. 1982. Precursor molecules of both human 5 S ribosomal RNA and transfer RNAs are bound by a cellular protein reactive with anti-La lupus antibodies. Cell 29: 149-159.

Rosado, I.V., Dez, C., Lebaron, S., Caizergues-Ferrer, M., Henry, Y., and de la Cruz, J. 2007. Characterization of Saccharomyces cerevisiae Npa2p (Urb2p) reveals a low-molecular-mass complex containing Dbp6p, Npa1p (Urb1p), Nop8p, and Rsa3p involved in early steps of 60 S ribosomal subunit biogenesis. Mol. Cell. Biol. 27: 1207-1221.

Saveanu, C., Bienvenu, D., Namane, A., Gleizes, P.E., Gas, N., Jacquier, A., and Fromont-Racine, M. 2001. Nog2p, a putative GTPase associated with pre-60S subunits and required for late 60S maturation steps. EMBO J. 20: 6475-6484.

Saveanu, C., Namane, A., Gleizes, P.E., Lebreton, A., Rousselle, 
J.C., Noaillac-Depeyre, J., Gas, N., Jacquier, A., and Fromont-Racine, M. 2003. Sequential protein association with nascent 60 S ribosomal particles. Mol. Cell. Biol. 23: 44494460.

Schaper, S., Fromont-Racine, M., Linder, P., de la Cruz, J., Namane, A., and Yaniv, M. 2001. A yeast homolog of chromatin assembly factor 1 is involved in early ribosome assembly. Curr. Biol. 11: 1885-1890.

Spahn, C.M.T., Beckmann, R., Eswar, N., Penczek, P.A., Sali, A., Blobel, G., and Frank, J. 2001. Structure of the 80S ribosome from Saccharomyces cerevisiae-tRNA-ribosome and subunit-subunit interactions. Cell 107: 373-386.

Spierer, P. and Zimmermann, R.A. 1978. Stoichiometry, cooperativity, and stability of interactions between 5S RNA and proteins L5, L18, and L25 from the 50S ribosomal subunit of Escherichia coli. Biochemistry 17: 2474-2479.

Steitz, J.A., Berg, C., Hendrick, J.P., La Branche-Chabot, H., Metspalu, A., Rinke, J., and Yario, T. 1988. A 5S rRNA/L5 complex is a precursor to ribosome assembly in mammalian cells. J. Cell Biol. 106: 545-556.

Tsay, Y.F., Shankweiler, G., Lake, J., and Woolford Jr., J.L. 1994. Localization of Saccharomyces cerevisiae ribosomal protein L16 on the surface of 60 S ribosomal subunits by immunoelectron microscopy. J. Biol. Chem. 269: 7579-7586.

Van Ryk, D.I., Lee, Y., and Nazar, R.N. 1992. Unbalanced ribosome assembly in Saccharomyces cerevisiae expressing mutant 5S rRNAs. J. Biol. Chem. 267: 16177-16181.

Viel, A., le Maire, M., Philippe, H., Morales, J., Mazabraud, A., and Denis, H. 1991. Structural and functional properties of thesaurin a (42Sp50), the major protein of the $42 \mathrm{~S}$ particles present in Xenopus laevis previtellogenic oocytes. J. Biol. Chem. 266: 10392-10399.

Warner, J.R. and Soeiro, R. 1967. Nascent ribosomes from HeLa cells. Proc. Natl. Acad. Sci. 58: 1984-1990.

Wehner, K.A. and Baserga, S.J. 2002. The $\sigma^{70}$-like motif: A eukaryotic RNA binding domain unique to a superfamily of proteins required for ribosome biogenesis. Mol. Cell 9: 329339.

West, M., Hedges, J.B., Chen, A., and Johnson, A.W. 2005. Defining the order in which Nmd3p and Rpl10p load onto nascent 60S ribosomal subunits. Mol. Cell. Biol. 25: 3802-3813.

Woolford Jr., J.L. and Warner, J.R. 1991. The ribosome and its synthesis, In The molecular and cellular biology of the yeast Saccharomyces: Genome dynamics, protein synthesis, and energetics. (eds. J.R. Broach et al.), pp. 587-626. Cold Spring Harbor Laboratory Press, Cold Spring Harbor, NY.

Xue, Y., Bai, X., Lee, I., Kallstrom, G., Ho, J., Brown, J., Stevens, A., and Johnson, A.W. 2000. Saccharomyces cerevisiae RAI1 (YGL246c) is homologous to human DOM3Z and encodes a protein that binds the nuclear exoribonuclease Ratlp. Mol. Cell. Biol. 20: 4006-4015.

Yao, W., Roser, D., Kohler, A., Bradatsch, B., Bassler, J., and Hurt, E. 2007. Nuclear export of ribosomal 60 S subunits by the general mRNA export receptor Mex67-Mtr2. Mol. Cell 26: 51-62.

Yoo, C.J. and Wolin, S.L. 1994. La proteins from Drosophila melanogaster and Saccharomyces cerevisiae: A yeast homo$\log$ of the La autoantigen is dispensable for growth. Mol. Cell. Biol. 14: 5412-5424.

Yu, R.S. and Wittmann, H.G. 1973. The sequence of steps in the attachment of 5-S RNA to cores of Escherichia coli ribosomes. Biochim. Biophys. Acta 324: 375-385.

Zinker, S. and Warner, J.R. 1976. The ribosomal proteins of Saccharomyces cerevisiae. Phosphorylated and exchangeable proteins. J. Biol. Chem. 251: 1799-1807. 


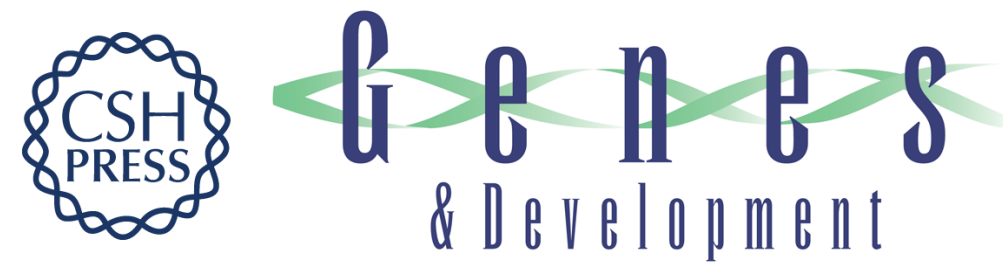

\section{Assembly factors Rpf2 and Rrs1 recruit 5S rRNA and ribosomal proteins rpL5 and rpL11 into nascent ribosomes}

Jingyu Zhang, Piyanun Harnpicharnchai, Jelena Jakovljevic, et al.

Genes Dev. 2007, 21:

Access the most recent version at doi:10.1101/gad.1569307

Supplemental http://genesdev.cshlp.org/content/suppl/2007/09/28/21.20.2580.DC1
Material

References This article cites 61 articles, 32 of which can be accessed free at:

http://genesdev.cshlp.org/content/21/20/2580.full.html\#ref-list-1

License

Email Alerting

Receive free email alerts when new articles cite this article - sign up in the box at the top

Service

right corner of the article or click here.

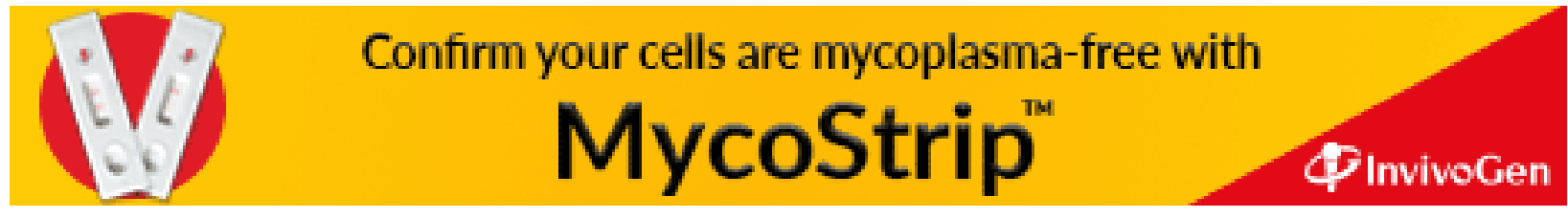

